\title{
Healing of Aneurysm after Treatment Using Flow Diverter Stent : Histopathological Study in Experimental Canine Carotid Side Wall Aneurysm
}

\author{
Jong Young Lee, ${ }^{1}$ Young Dae Cho, ${ }^{2}$ Hyun-Seung Kang, ${ }^{3}$ Moon Hee Han ${ }^{4}$ \\ Department of Neurosurgery, Hallym University Gangdong Sacred Heart Hospital, Hallym University College of Medicine, Seoul, Korea \\ Department of Radiology, ${ }^{2}$ Seoul National University Hospital, Seoul National University College of Medicine, Seoul, Korea \\ Department of Neurosurgery, ${ }^{3}$ Seoul National University Hospital, Seoul National University College of Medicine, Seoul, Korea \\ Department of Neurosurgery, ${ }^{4}$ Veterans Health Service Medical Center, Seoul, Korea
}

Objective : Despite widespread use of flow diverters (FDs) to treat aneurysms, the exact healing mechanism associated with FDs remains poorly understood. We aim to describe the healing process of aneurysms treated using FDs by demonstrating the histopathologic progression in a canine aneurysm model.

Methods : Twenty-one side wall aneurysms were created in common carotid artery of eight dogs and treated with two different FDs. Angiographic follow-ups were done immediately after placement of the device, 4 weeks and 12 weeks. At last follow-up, the aneurysm and the device-implanted parent artery were harvested.

Results : Histopathologic findings of aneurysms at 4 weeks follow-up showed intra-aneurysm thrombus formation in laminating fashion, and neointimal thickening at the mid-segment of aneurysm. However, there are inhomogenous findings in aneurysms treated with the same type of FD showing same angiographic outcomes. At 12 weeks, aneurysms of complete and near-complete occlusion revealed markedly shrunken aneurysm filled with organized connective tissues with thin neointima. Aneurysms of incomplete occlusion at 12 weeks showed small amount of organized thrombus around fringe neck and large empty space with thick neointmal formation. Neointimal thickness and diameter stenosis was not significantly different between the groups of FD specification and follow-up period.

Conclusion : Intra-aneurysmal thrombus formation and organization seem to be an important factor for the complete occlusion of aneurysms treated using the FD. Neointimal formation could occur along the struts of the FD independently of intra-aneurysmal thrombus formation. However, neointimal formation could not solely lead to complete aneurysm healing.

Key Words : Canine · Aneurysm · Flow · Diversion · Stents · Histology.

\footnotetext{
- Received : March 11, 2019 •Revised : April 17, 2019 •Accepted : April 22, 2019

- Address for reprints : Moon Hee Han

Department of Neurosurgery, Veterans Health Service Medical Center, 53 Jinhwangdo-ro 61-gil, Gangdong-gu, Seoul 05368, Korea Tel : +82-2-2225-1363, Fax : +82-2-2225-4152, E-mail : hanmoonhee@gmail.com, ORCID : https://orcid.org/0000-0001-5476-3482
} 


\section{INTRODUCTION}

Flow diverters (FDs) have been used widely for the treatment of intracranial with good clinical results ${ }^{1,3,6,7,9,10,16,24,29)}$. However, the exact healing mechanism of the flow diverter remains poor. To evaluate the healing process, numerous exvivo and/or in-vivo experimental studies have been focused on relationship between degree of hemodynamic alteration induced by different type of FDs and their efficacy ${ }^{2,8,12,15,25)}$. Some in-vivo experimental studies demonstrated aneurysm healing process, emphasizing neointima formation by using various biomarkers ${ }^{13,20,21)}$. Even though these data provide useful information to understand aneurysm healing mechanism with FDs, there are some limitations to our comprehension of the overall aneurysm healing process. The aim of the present study was to describe the healing process of aneurysms treated using an FD by demonstrating the intuitive histopathologic findings compared with angiographic outcomes in a canine side wall aneurysm model.

\section{MATERIALS AND METHODS}

\section{Animal experiments}

All experimental protocols were approved by the Institutional Animal Care and Use Committee (IACUC) of Seoul National University Hospital (SNUH-IACUC No. 11-0001), and conducted in accordance with the animal experiment guidelines. Twenty-one wide-necked, side-wall saccular aneurysms using venous pouch were made in eight morphologically normal male, mongrel dogs of similar size and weight (28$32 \mathrm{~kg}$ ). Operations were performed under sterile conditions. After induction with intramuscular injection of $15 \mathrm{mg} / \mathrm{kg}$ of Zoletil (zolazepam and tiletamine; Virbac AH, Fort Worth, TX, USA) and $10 \mathrm{mg} / \mathrm{kg}$ of Rompun (xylazine; Bayer Animal Health GmbH, Pfullingen, Germany), general endotracheal anesthesia was maintained using $1 \%$ to $3 \%$ inhaled isoflurane (Forane; Choongwae Pharma, Seoul, Korea) throughout the procedure. After completion of survival procedures, the animals were given $2 \mathrm{mg} / \mathrm{kg}$ of subcutaneous Ketoprofen (Ketoprofen; UniBiotech, Seoul, Korea) as analgesic, anti-inflammatory and antipyretic immediately after surgery, and twice a day for 3 days postoperatively. Detailed procedures for aneurysm creation have been described elsewhere ${ }^{14,22)}$. In brief, we prepared 6-8 $\mathrm{mm}$ sections of the vein. One end of each venous segment was ligated using 3-0 black silk. The open end of the prepared venous segment was sewn along the edge of the arteriotomy (approximately $10 \mathrm{~mm}$ in length) using 7-0 Prolene sutures (Ethicon, Inc., Somerville, NJ, USA). All aneurysms were checked using digital subtraction angiography (DSA) at 1 and 4 weeks after aneurysm creation using mobile C-arm system (BV Pulsera, Philips Medical System, the Netherland). We used aneurysm models as experimental subjects when the patency of the intra-aneurysmal and parent arterial flow was confirmed by 4 -week DSA.

\section{Flow diverter device}

The devices were constructed of a 0.002-inch nitinol and platinum wires, and were self-expanding in nature. Stents (NOD stent; Access Point Technologies LLC, Roger, MN, USA) were manufactured using an automated braiding machine. The stent consisted of two different parts; a central portion, middle $1 / 3$ of stent length, and peripheral portion, proximal and distal $1 / 3$ of stent with smoother, wider interstices. (Fig. 1). In this experimental study, two different flow diverters were used, i.e., 32- and 48-strand braided stent, which have different profiles at nominal diameter (Table 1). The stent profile was described using the porosity, which is defined as the ratio of the metal free surface area to the total surface area, the pore per inch (PPI), which is defined as the number of pores per unit length of the stent and represent the pore density, and pore size, which is defined as the area of a pore $\left(\mathrm{mm}^{2}\right)$. All two flow diverters had an open device diameter of $4 \mathrm{~mm}$ or $5 \mathrm{~mm}$, and an open device length of $30 \mathrm{~mm}$. Compared with commercially available flow diverter stent, e.g., pipeline embolization device (PED; ev3 Endovascular Inc/Covidien, Plymouth, MN, USA), and Silk (Balt Extrusion, Montmorency, France), newly designed FD shows lower porosity and PPI at the midsegment of stent $(77.2 \pm 2.9 \%, 70.4 \pm 1 \%, 64.1 \%$ of 32 -strand FD, and $55.4 \%$ of 48 -strand FD; 128, 144, 61 of 32-strand FD, and 55.4 of 48 -strand FD, respectively). Mid-segment pore size of newly designed FD stent is similar to PED and silk FD stent $\left(0.04 \mathrm{~mm}^{2}\right.$ of 32-strand FD, $0.03 \mathrm{~mm}^{2}$ of 48 -strand FD, $0.02-$ $0.05 \mathrm{~mm}^{2}$, and $0.01-0.03 \mathrm{~mm}^{2}$, respectively). The self-expanding FD is packaged pre-loaded into the 0.49 microcatheter delivery system in its elongated form with $3 \mathrm{~cm}$ tip guide microwire. 


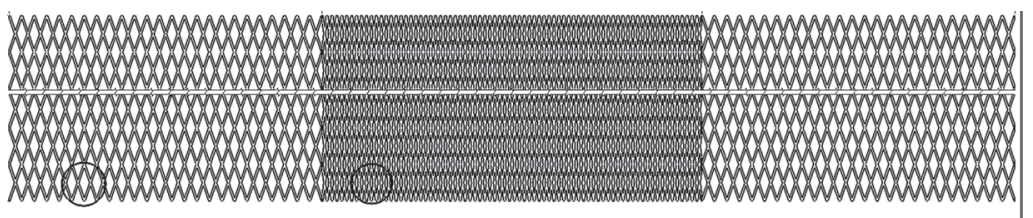

(A)

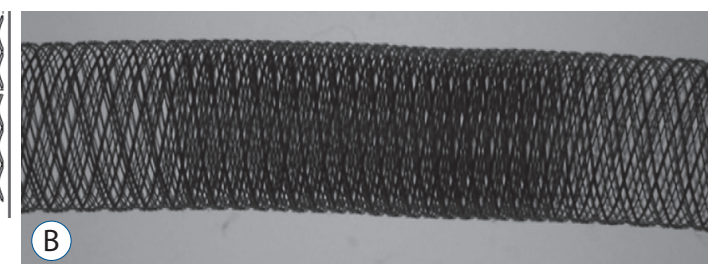

B

Fig. 1. Schematic diagram (A) and image (B) of the flow diverter configuration. This stent (B) contains 32 nitinol wires, 4 of which are equipped with platinum wire to enhance overall visibility. Mid segment of the stent has tight braiding distance.

Table 1. Profiles of flow diverters

\begin{tabular}{lcccccc}
\hline & \multicolumn{2}{c}{ 32-strand } & & \multicolumn{2}{c}{ 48-strand } \\
\cline { 2 - 3 } \cline { 5 - 6 } & Mid & End & & Mid & End \\
\hline Pore size $\left(\mathrm{mm}^{2}\right)$ & 0.13 & 0.04 & & 0.08 & 0.03 \\
Porosity (\%) & 64.1 & 80.3 & & 55.4 & 77.8 \\
PPI & 61 & 123 & & 76 & 153 \\
\hline PPI : pore per inch & & & & &
\end{tabular}

\section{Stent implantation}

After allowing sufficient time for the aneurysm to mature (approximately 4 weeks), FDs were implanted at the aneurysms. All treated animals had been orally given $50 \mathrm{mg}$ of aspirin and $37.5 \mathrm{mg}$ of clopidogrel orally once a day beginning 7 days before the procedure and continuing until 12 weeks after the procedure. The same anesthesia as described for aneurysm creation was employed for stent implantation. After surgical exposure of the right common femoral artery using sterile technique, a $6 \mathrm{~F}$ vascular sheath was introduced into the vessel. Heparin $(100 \mathrm{U} / \mathrm{kg})$ was administered intravenously. Under fluoroscopic guidance, a $6 \mathrm{~F}$ guiding catheter (Envoy, Cordis Neurovascular Systems, Miami Lakes, FL, USA) was advanced into the common carotid artery. A roadmap image was then obtained to identify the exact location of the aneurysm neck. The pre-loaded FD system was advanced until the mid-segment covered the aneurysm neck. To achieve optimal wall apposition of FD and flow modification augmentation, the pre-loaded FD is released by pushing the transport wire while gently retrieving the microcatheter at the same time. Next, the whole system was appropriately pushed up. These maneuvers are repeated during the procedure to achieve optimal deployment. The choice of device between two different FDs to be implanted in any given animal was solely dependent on the study schedule and was otherwise arbitrary.

\section{Angiographic evaluation, follow-up and sacrifice of the animals}

Analysis of aneurysm size was performed using linear measurements, i.e., maximal diameter and dome-to-neck (DN) ratio, obtained using the working projection and geometric comparison with reference vessel or a $10 \mathrm{~mm}$ round metal marker $^{11)}$.

To evaluate the immediate outcomes of aneurysms treated with FD, intra-aneurysmal flow modification was classified as complete stasis (if no contrast media entered the aneurysm following deployment of the FD), significant flow reduction (if contrast stagnation was seen within the aneurysm in the late venous phase of the angiographic series), or slow flow (if the contrast circulation within the aneurysm became slower but without contrast stagnation in the late venous phase images) ${ }^{27}$.

Animals were followed-up at 4 weeks ( $\mathrm{n}=13$, five animals) and 12 weeks ( $\mathrm{n}=8$, three animals) after the FD implantation. Follow-up angiograms were acquired via transfemoral access as described above. Immediate and follow-up angiographic outcomes were analyzed using a five-point grading scheme as follows : grade 0 , no intra-aneurysmal flow change; grade I, residual aneurysmal contrast filling $\geq 50 \%$; grade II, residual aneurysmal contrast filling $<50 \%$; grade III, residual contrast filling confined to the neck region; grade IV, no residual contrast filling ${ }^{17)}$. Two observers analyzed all angiographic data, and consensus was reached by means of discussion in cases of discrepancy.

Under deep anesthesia inducted with ketamine $(50 \mathrm{mg} / \mathrm{kg}$ ) and Rompun $(10 \mathrm{mg} / \mathrm{kg})$, the animal was euthanized with an intravenous administration of potassium chloride after final angiographic follow-up. The aneurysm-parent artery complex was then explanted and flushed with normal saline and 10\% formalin. 
Table 2. Aneurysm description by duration in 32-strand and 48-strand stent

\begin{tabular}{lcccccccc}
\hline Time (weeks) & $\begin{array}{c}\text { 32-strand } \\
\text { width }(\mathbf{m m})\end{array}$ & $\begin{array}{c}\text { 48-strand } \\
\text { width }(\mathbf{m m})\end{array}$ & $\begin{array}{c}\text { 32-strand } \\
\text { neck }(\mathbf{m m})\end{array}$ & $\begin{array}{c}\text { 48-strand } \\
\text { neck }(\mathbf{m m})\end{array}$ & $\begin{array}{c}\text { 32-strand } \\
\text { depth }(\mathbf{m m})\end{array}$ & $\begin{array}{c}\text { 48-strand } \\
\text { depth }(\mathbf{m m})\end{array}$ & $\begin{array}{c}\text { 32-strand } \\
\text { DN ratio }\end{array}$ & $\begin{array}{c}\text { 48-strand } \\
\text { DN ratio }\end{array}$ \\
\hline 4 & $9.4 \pm 0.8$ & $10.1 \pm 0.6$ & $8.8 \pm 0.7$ & $9.0 \pm 0.5$ & $8.0 \pm 0.9$ & $8.9 \pm 0.7$ & $0.9 \pm 0.1$ & $1.0 \pm 0.1$ \\
12 & $8.8 \pm 0.9$ & $9.4 \pm 0.9$ & $8.1 \pm 0.7$ & $9.0 \pm 0.7$ & $8.0 \pm 1.1$ & $6.3 \pm 1.1$ & $1.0 \pm 0.1$ & $0.7 \pm 0.1$ \\
$p$-value & \multicolumn{2}{c}{0.92} & & 0.57 & & 0.19 & 0.09 \\
\hline
\end{tabular}

DN : dome-to-neck

\section{Tissue processing}

The formalin-fixed tissue samples were processed through a graded series of ethanol, xylene, and were embedded in methyl-methacrylate. Three representative cross sections per stented segment (proximal, middle, and distal) for the 14 early aneurysms and one longitudinal section for the seven late aneurysms were taken from the block at approximately $600-\mu \mathrm{m}$ interval, polished down to $6 \mu \mathrm{m}$, and stained with hematoxilin-eosin (HE) stain.

Morphometric measurements were performed as follows : thickness of neointima, distance between the outer surface of stent strut and the luminal border at the thickest area; neolumen, distance from luminal border to luminal border; former vessel lumen, distance from the outside of a strut to the opposite outside of the strut across the vessel diameter; diameter stenosis $=($ neolumen $/$ former lumen $) \times 100$ at the narrowest neoluminal area.

\section{Statistical analysis}

Angiographic outcomes were compared between the two different FD groups, 32-strand stent and 48-strand stent, to evaluate the degree of flow diversion. We analyzed angiographic aneurysmal dimensions using a 2-way analysis of variance (ANOVA; stent ${ }^{\star}$ follow-up duration).

The chi-square test was used to compare the frequency distributions of categorical variables between the study groups. Continuous variables were analyzed by the Mann-Whitney $U$ test. Probability values of less than 0.05 were regarded as statistically significant. All statistical analyses were performed with SPSS version 17.0 software for Windows (SPSS Inc., Chicago, IL, USA).

\section{RESULTS}

\section{Angiographic findings}

All of surgically created 21 aneurysms remained patent during a follow-up period of 4 weeks. Two-way ANOVA revealed no interaction between the two groups for width, neck size, depth, or DN ratio of the aneurysm (Table 2). If the main effect "stent" was examined and all time points were grouped together, there were no differences in width $(9.1 \pm 0.6 \mathrm{~mm}$ vs. $9.7 \pm 0.5 \mathrm{~mm} ; p=0.41)$, neck size $(8.5 \pm 0.5 \mathrm{~mm}$ vs. $9.0 \pm 0.5 \mathrm{~mm}$; $p=0.44)$, depth $(8.0 \pm 0.7 \mathrm{~mm}$ vs. $7.6 \pm 0.6 \mathrm{~mm} ; p=0.66)$, or $\mathrm{DN}$ ratio $(0.9 \pm 0.1 \mathrm{~mm}$ vs. $0.9 \pm 0.1 \mathrm{~mm} ; p=0.38)$ for aneurysms treated with 32-strand vs. 48-strand stent, respectively.

In all cases, the delivery and deployment of the devices were successful without periprocedural complications. Table 3 summarizes angiographic outcomes. After placement of the FD, control angiogram showed significant flow reduction in 1laneurysms and slow flow in 10 aneurysms. There was no difference in flow modification between the two groups ( $p=0.67 ; 2$-tailed Fisher's Exact test). In accordance with the five-point grading scheme, overall occlusion rates of grade $0, \mathrm{I}$, and II were noted in nine (42.9\%), 10 (47.6\%), and two (9.5\%) of 21 aneurysms, respectively. There was no difference in the immediate angiographic occlusion rate between the two groups ( $p=0.32$; 2-tailed Fisher's Exact test).

At 4 weeks, follow-up angiography revealed two (22.2\%) vs. 0 of grade 0 , three $(33.3 \%)$ vs. 0 of grade I, three $(33.3 \%)$ vs. five $(41.7 \%)$ of grade II, 0 vs. three $(25.0 \%)$ of grade III, and one $(11.1 \%)$ vs. four $(33.3 \%)$ of grade IV occlusion of aneurysms treated with 32-strand versus 48-strand stents, respectively. Aneurysms treated with the 48-strand FD showed higher occlusion rate compared with aneurysms treated with the 32-strand FD ( $p$ for trend=0.009). Flow modification was not associated with aneurysmal occlusion rate at 4-week follow-up ( $p$ for trend=0.18). On the other hand, a higher grade immediate angiographic occlusion rate was significantly asso- 
Table 3. Angiographic outcomes in aneurysms treated with flow diverter

\begin{tabular}{|c|c|c|c|c|}
\hline Group and An. No. & Flow modification & Immediate outcome & At 4 weeks & At 12 weeks \\
\hline \multicolumn{5}{|l|}{ 32-strand } \\
\hline 1 & Slow flow & 1 & 1 & $\|$ \\
\hline 2 & Slow flow & 0 & । & । \\
\hline 3 & Significant flow reduction & 0 & । & । \\
\hline 4 & Significant flow reduction & 0 & 0 & 0 \\
\hline 8 & Slow flow & । & $\|$ & \\
\hline 9 & Significant flow reduction & I & $\|$ & \\
\hline 15 & Slow flow & I & IV & \\
\hline 16 & Significant flow reduction & 0 & $\|$ & \\
\hline 21 & Significant flow reduction & 0 & 0 & \\
\hline \multicolumn{5}{|l|}{ 48-strand } \\
\hline 5 & Slow flow & I & IV & \\
\hline 6 & Slow flow & 1 & $\|$ & \\
\hline 7 & Significant flow reduction & 0 & $\|$ & \\
\hline 10 & Slow flow & 0 & $\|$ & \\
\hline 11 & Slow flow & 0 & $\|$ & IV \\
\hline 12 & Slow flow & 1 & $\|$ & IV \\
\hline 13 & Significant flow reduction & 1 & IV & IV \\
\hline 14 & Significant flow reduction & 0 & III & III \\
\hline 17 & Significant flow reduction & 1 & IV & \\
\hline 18 & Slow flow & । & III & \\
\hline 19 & Slow flow & $\|$ & IV & \\
\hline 20 & Slow flow & $\|$ & III & \\
\hline
\end{tabular}

An. : aneurysm

ciated with higher grade occlusion rate at 4-week follow-up ( $p$ for trend $=0.008$ ).

At 12 weeks, follow-up angiograms revealed one $(25.0 \%)$ vs. 0 of grade 0 , two (50.0\%) vs. 0 of grade I, one (25.0\%) vs. 0 of grade II, 0 vs. one (25.0\%) of grade III, and 0 vs. three $(75.0 \%)$ of grade IV occlusion of aneurysms treated with 32-strand versus 48-strand stents, respectively. Aneurysms treated with the 48-strand FD showed a higher occlusion rate compared with aneurysms treated with the 32-strand FD ( $p$ for trend $=0.029$ ). Flow modification and immediate angiographic occlusion rate was not associated with the occlusion rate at 12week follow-up ( $p$ for trend $=0.59$ and 0.13 , respectively).

\section{Histopathologic findings}

At 4 weeks, intra-aneurysmal histopathologic findings of completely occluded aneurysms (grade IV) were variable.
Coronal sections of aneurysm 5 show that the aneurysmal sac was filled with concentrically laminated thrombi of various stages of organization (Fig. 2C-E). The thrombi of aneurysm 19 revealed as irregular, ill-defined laminations primarily composed of organized thrombus (Fig. 2F). Midline longitudinal sections of aneurysm 15 and 17 showed significantly shrunken aneurysmal sac, and small area of organized thrombus and attenuated cellular matrix with thick neointima formation (Fig. 2G). Aneurysms with near occlusion (grade III) also demonstrate diverse findings (Fig. 3A and C). A midline longitudinal section of aneurysm 20 showed that the aneurysmal sac was filled with laminated thrombus of various stages of organization (Fig. 3B). Aneurysm 18 was shrunken in its size, and fresh blood clot surrounded by organized thrombus was present. The distance between stent struts was relatively wide at the segment proximal to the fresh blood clot (arrow- 

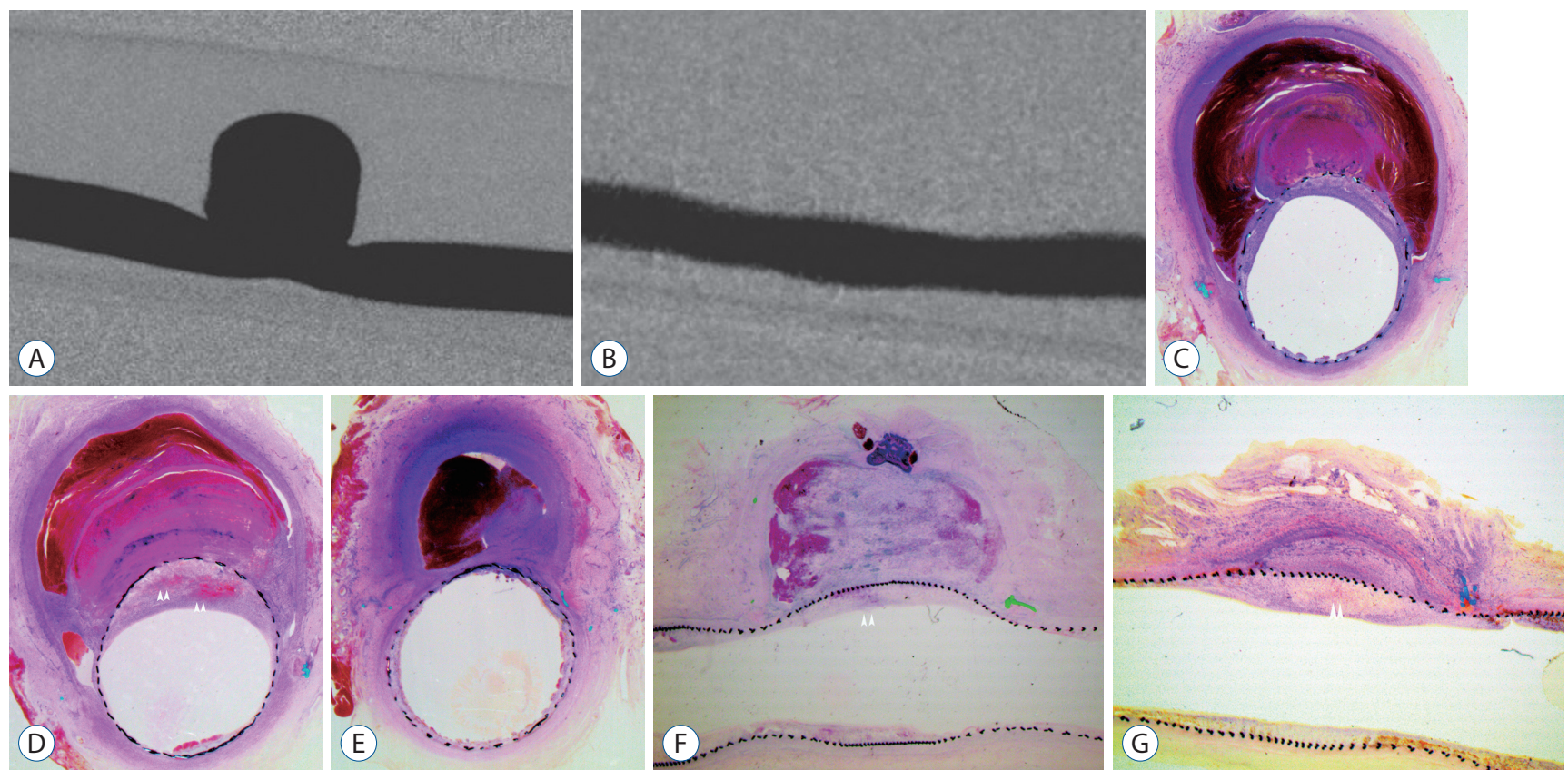

Fig. 2. A : Pre-procedural common carotid arteriogram shows a large, wide-necked side wall aneurysm (aneurysm 5) B : Four weeks FU angiograph of aneurysm 5 shows complete occlusion of aneurysm. C-E : Photomicrographs of aneurysm 5. Proximal (C), mid (D), and distal (E) segment of coronal sections shows multi-staged thrombus formation in concentrically laminated fashion (C-E : H-E stain, $\times 40)$. F : Midline longitudinal section of aneurysm 19 shows the thrombi revealed as irregular, ill-defined laminations which are mainly composed with organized thrombus (H-E stain, $\times 40)$. G : Midline longitudinal section of aneurysm 15 shows a significantly shrunken aneurysmal sac, and small area of organized thrombus and attenuated cellular matrix with neointimal hyperplasia around an aneurysmal neck (H-E stain, $\times 40$ ). Neointimal hyperplasia was shown in the mid-segment of aneurysm, and it contained organized blood clot (arrowheads in D and F). FU : follow-up.

heads, Fig. 3D) compared with other segments. The aneurysmal sacs of incompletely occluded aneurysms (grade 0-II) were usually filled with multi-staged thrombus primarily composed of fresh blood clot with or without a small empty space (Fig. 3E and G). A midline longitudinal section of aneurysm 16 showed a small amount of organized thrombus formation around stent struts with an intimal defect (Fig. $3 \mathrm{H}$ and I).

At 12 weeks, grade IV aneurysms were significantly shrunken, and histopathology showed a small area of organized thrombus and attenuated cellular matrix with a thin neointima (Fig. 4A and B). Grade III aneurysms was also was significantly shrunken in its size, and histopathology shows small area of organized thrombus and attenuated cellular matrix with small amount of unorganized blood clot (Fig. 4C and D). Incompletely occluded aneurysms (grade $0-$ II) most commonly showed empty sacs with small amounts of variably organized thrombus formation at the fringe neck formed between the aneurysmal wall and stent struts in coronal sections with large empty space within the aneurysmal sac (Fig. 4E-H).

Neointimal thickness and diameter stenosis was not signifi- cantly different between 4 -week and 12-week follow-up, but showed a decreasing tendency after 12 weeks in completely occluded aneurysms $(0.99 \pm 0.57 \mathrm{~mm}$ vs. $0.53 \pm 0.14 \mathrm{~mm}$, $p=0.16 ; 29.6 \pm 8.36 \mathrm{~mm}$ vs. $13.1 \pm 3.54 \mathrm{~mm}, p=0.69)$. In contrast, neointimal thickness and diameter stenosis showed an increasing trend after 12 weeks in near complete $(0.84 \pm 0.34 \mathrm{~mm}$ vs. $0.97 \pm 0.43 \mathrm{~mm}, p=0.78 ; 17.2 \pm 6.52 \mathrm{~mm}$ vs. $19.0 \pm 26.1 \mathrm{~mm}$, $p=0.91)$ and incomplete $(0.94 \pm 0.29 \mathrm{~mm}$ vs. $1.13 \pm 0.59 \mathrm{~mm}$, $p=0.49 ; 23.1 \pm 5.03 \mathrm{~mm}$ vs. $39.9 \pm 7.16 \mathrm{~mm}, p=0.16$ ) occluded aneurysms. Neointimal thickness and diameter stenosis between the groups treated with 32-strand and 48-strand FDs were not significantly different $(1.01 \pm 0.37 \mathrm{~mm}$ vs. $0.81 \pm 0.39 \mathrm{~mm}$, $p=0.82 ; 30.2 \pm 11.1 \mathrm{~mm}$ vs. $17.8 \pm 8.31 \mathrm{~mm}, p=0.34$ ).

\section{DISCUSSION}

According to various experimental data on intra-aneurysmal hemodyniamics, FDs disrupt the vortical flow of aneurysms, and decreases the inflow rate ${ }^{4,23}$. As a result, intra-an- 

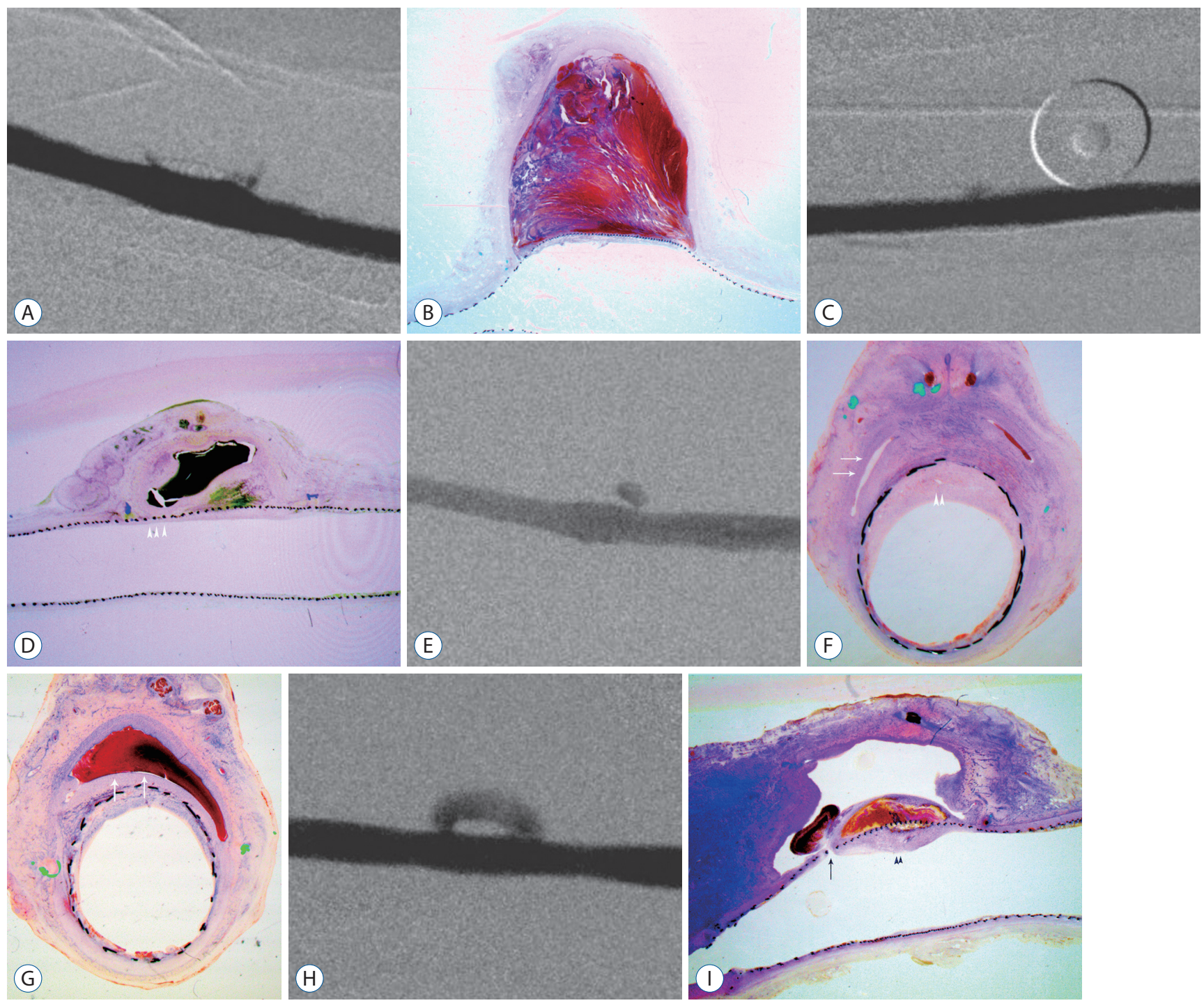

Fig. 3. A : Four weeks FU angiograph of aneurysm 20 shows near complete occlusion of aneurysm (grade III). B : Midline longitudinal section of aneurysm 20 shows that aneurysmal sac was filled with laminated thrombus various stages of organization (H-E stain, $\times 40)$. C : Four weeks FU angiograph of aneurysm 18 shows near complete occlusion of aneurysm (grade III). D : Midline longitudinal section of aneurysm 18 shows that it was shrunken in its size, and fresh blood clot surrounded by organized thrombus was shown. Distant between stent struts was relatively wide at the segment proximity to the fresh blood clot (arrowheads in D) compared with other segments. E : Four weeks FU angiograph of aneurysm 9 shows incomplete occlusion of aneurysm (grade II). F and G : Photomicrographs of aneurysm 9. Mid (F) and distal (G) segment of coronal sections shows that the aneurysmal sac was filled with multi-staged thrombus mainly composed of fresh blood clot (arrows in $\mathrm{G}$ ) with small empty space (arrows in F) (G and $\mathrm{F}: \mathrm{H}$-E stain, $\times 40$ ). $\mathrm{H}$ : Four weeks FU angiograph of aneurysm 16 shows incomplete occlusion of aneurysm (grade II). I : Midline longitudinal section of Aneurysm 16 shows small amount of organized thrombus along the stent strut with intimal defect (arrow in I) (H-E stain, $\times 40)$. Small amount of fresh blood clot was show adjacent to the intimal defect with large empty space of the aneurysmal sac. Neointimal hyperplasia was shown in the midsegment of aneurysm, and it contained organized blood clot (arrowheads in F and I). FU : follow-up.

eurysmal thrombosis is induced by the modified local hemodynamic conditions. Especially, sidewall aneurysm hemodynamic studies revealed that the intra-aneurysmal vortex after FD implantation starts at the distal neck and flows along the aneurysmal wall, with flow stagnation more prominent at the proximal segment of the aneurysm and proximal to the mid-segment of the neck. Our histopathologic findings support this hypothesis. Intra-aneurysmal thrombi were formed in a laminated pattern in completely and near-completely occluded aneurysms at 4-week follow-up, with a various stages of organization (Figs. 2 and 3). The presence of a laminated thrombus implies thrombosis at the site of blood flow, which 

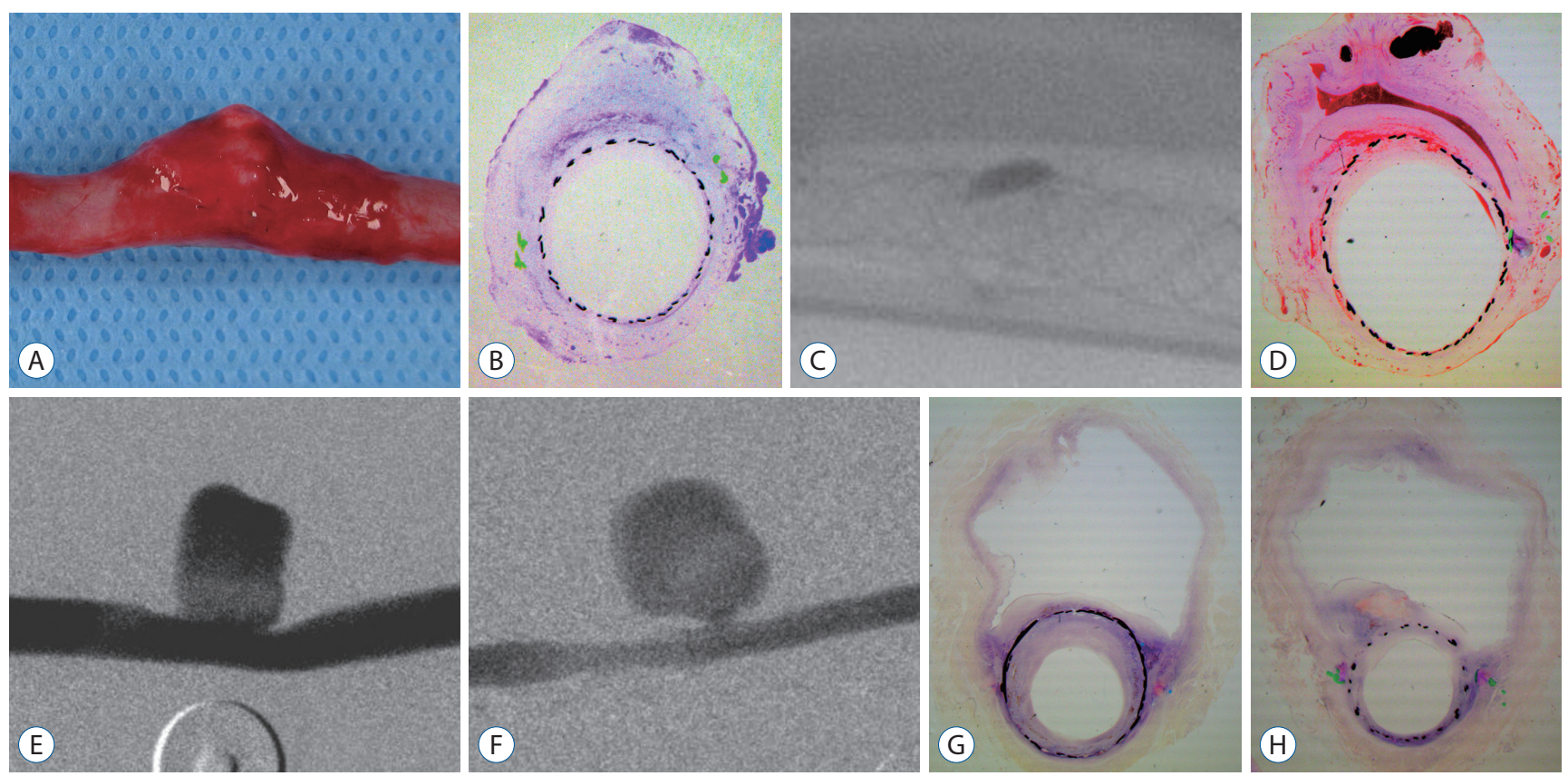

Fig. 4. A : Gross inspection of completely occluded aneurysm 13 (grade IV). It is significantly shrunken in its size. B : Photomicrographs of mid-segment of coronal sections (aneurysm 13) (H-E stain, $\times 40$ ). Aneurysmal sac was significantly shrunken, and histopathology shows small area of organized thrombus and attenuated cellular matrix with thin neointima. C : Native plane radiograph of aneurysm 14 obtained immediately after 12-week followup angiography. It shows small amount of contrast stagnation at the aneurysmal neck (grade III). D : Photomicrographs of aneurysm 14. Mid-segment of coronal section shows significantly shrunken aneurysmal sac, and small area of sharp, crescentic fresh blood clot and attenuated cellular matrix with thin neointima (H-E stain, $\times 40$ ). E : Pre-procedural common carotid arteriogram shows a large, wide-necked side wall aneurysm (aneurysm 4). F : Twelveweeks follow-up angiograph of aneurysm 4 shows incomplete occlusion of the aneurysm, and neointimal formation with intimal defect at the distal segment of aneurysmal neck. $\mathrm{G}$ and $\mathrm{H}$ : Photomicrographs of aneurysm 4. Proximal (G) and mid (H) segment of coronal sections shows empty sac with small amount of variably organized thrombus formation at the fringe neck formed between aneurysmal wall and stent struts with intimal hyperplasia at proximal segment of aneurysm ( $\mathrm{G}$ and $\mathrm{F}: \mathrm{H}-\mathrm{E}$ stain, $\times 40)$.

means thrombus formation progresses gradually along the direction of flow ${ }^{19}$. Fig. 3B showed an intra-aneurysmal thrombus with lamination, and the pattern of lamina resembles the flow direction of the intra-aneurysmal vortex after FD deployment. Fig. 2C and D showed relatively more organized thrombus was formed around proximal- and mid-segment of neck area. Histopathologic findings of incompletely occluded aneurysms revealed that organized thrombus was present at the neck area or a fringe neck formed between the aneurysmal wall and stent struts, with fresh blood clot or empty space in the aneurysmal dome (Figs. 3I, 4G and H). These locations where more organized thrombi were observed would represent hemodynamically inert areas immediately after FD deployment, as previously demonstrated in ex-vivo experimental data ${ }^{27,28}$. According to these findings, we hypothesized that each layer of variously organized thrombi would be gradually formed at different time along the newly induced intra-aneurysmal flow associated with the FD.
We assumed that relatively similar pattern of intra-aneurysmal histopathologic findings in aneurysms treated using a same specification of FD at specific follow-up period. However, various degrees and patterns of intra-aneurysmal thrombus formation with different sized aneurysm sacs were found among aneurysms treated using the same type of FD and showing the same angiographic outcomes at a specific followup period. Histopathologic findings of aneurysm 18 and 16 show the proximity of fresh blood clot or neointimal defect with a small amount of fresh blood clot at the segment where the distance between stent struts was relatively wide (Fig. 3D and F). Although same specification of FD, various degree of compaction mesh could be achieved around the aneurysmal neck. And, it might attenuate the inertia-driven inflow jet and redirected it to the more proximal segment of the aneurysm. Active inflow remained through these segment might disrupt stable intra-aneurysmal thrombus formation. In addition, inherent thrombogenicity and degree of antiplatelet response 
might be associated with these histopathologic findings. Following intra-aneurysmal thrombus formation induced by the $\mathrm{FD}$, the thrombus is replaced by organized connective tissue. In sequence, this would lead to shrinkage of the aneurysm to various degrees $^{18)}$. Altered size and geometry of the aneurysm would lead to additional intra-aneurysmal hemodynamic changes. Heterogeneity of thrombogenicity and the antiplatelet response is well known ${ }^{26)}$, and this might also result in a greater or lesser degree of thrombus formation and organization. With regard to these findings, the angiographic outcome could not represent an exact aneurysm healing process after FD deployment.

Neointimal formation of variable thickness was observed in all aneurysms treated with FD, and there was no significant difference between the groups treated with two different FDs. Kadirvel et al. ${ }^{13)}$ demonstrated that endothelialization is exclusively derived from cells in the adjacent parent artery, and smooth muscle and endothelial cells grow over the struts of the device itself. Regardless of stent specification, it is supposed that struts of the FD act as scaffolds for neointimal formation. In addition, neointimal formation seems to be independent of intra-aneurysmal thrombus formation. Histopathologic findings of aneurysm 4, which shows grade 0 occlusion at 12-week follow-up, support this hypothesis. This aneurysm showed thick neointima formation with a large empty space in the intra-aneurysmal sac and a scant amount of intra-aneurysmal thrombus formation. Some authors suggested that neointimal formation is more important than intra-aneurysmal thrombus formation in the complete occlusion of aneurysms ${ }^{13,21)}$. In accordance with these findings, however, we hypothesize that neointimal formation is an independent process to intra-aneurysmal thrombus formation, and complete occlusion of an aneurysm could not be achieved without intra-aneurysmal thrombus formation.

\section{Limitations}

Our study has several limitations. First, we use canine venous pouch aneurysm models. Compared with elastase-induced arterial aneurysm model, it is less physiologically accurate $^{14)}$. However, currently available, various animal models present multiple weaknesses in term of implementation of real aneurysms of human ${ }^{5)}$. Using canine venous pouch aneurysm models, we were able to create relatively consistent-sized aneurysms that provided similar intra-aneurysmal hemodynamics.
In addition, the lack of spontaneous thrombosis or rupture ${ }^{28)}$ is valuable for evaluation of histopathologic changes within the aneurysms induced only by FDs without any unexpected events. Second, even though we recognize inherent thrombogenecity and the antiplatelet response as independent factors associated with aneurysmal healing, we could not evaluate these in the present study. Based on the dogs' weight, which was around $30 \mathrm{~kg}$, a half-dose of standard aspirin and clopidogrel, as prescribed for adult patients at our institute, was used. We suspect that our antiplatelet medication strategy for experimental animals might reflect our clinical practice. Because of the difference between species and heterogeneity of the antiplatelet response, however, our findings should be interpreted carefully. Finally, the number of aneurysms included in the study is limited, especially in the 12-week follow up group. Studies with a larger number of subjects are needed to confirm the trends observed in this experimental study. Despite these limitations, this study demonstrates the healing process of aneurysms treated using FDs by documenting histopathologic findings over time. By comparing angiographic and histopathologic findings, our results provide a useful schema for the healing process of aneurysm treated using FDs in clinical practice.

\section{CONCLUSION}

Intra-aneurysmal thrombus formation and organization is a gradually progressive sequence of events, influenced by the modified intra-aneurysmal flow induced by FD placement, subsequently leading to dynamic intra-aneurysmal hemodynamic changes affecting aneurysm healing. Among aneurysms showing the same angiographic outcomes at a specific follow-up period, various degrees and patterns of intra-aneurysmal thrombus formation with different sized aneurysm sacs were found. Neointimal formation could occur along the struts of the FD independently of intra-aneurysmal thrombus formation. However, neointima formation could not solely lead to complete aneurysm healing. Intra-aneurysmal thrombus formation and organization seems to be an important factor for the complete occlusion of aneurysms treated using a FD. 


\section{CONFLICTS OF INTEREST}

No potential conflict of interest relevant to this article was reported.

\section{AUTHOR CONTRIBUTIONS}

\author{
Conceptualization : MHH, HSK,JYL \\ Data curation : JYL, MHH \\ Formal analysis: JYL, MHH \\ Funding acquisition : $\mathrm{MHH}$ \\ Methodology : MHH, JYL \\ Project administration : MHH, JYL \\ Visualization : JYL, MHH \\ Writing - original draft : JYL, MHH \\ Writing - review \& editing : JYL, YDC, HSK, MHH
}

\section{- Acknowledgements}

This work was supported by a grant of the Korean health technology R\&BD Project, Ministry of Knowledge Economy, Republic of Kore (RBD-0901-034).

\section{References}

1. Arrese I, Sarabia R, Pintado R, Delgado-Rodriguez M : Flow-diverter devices for intracranial aneurysms: systematic review and meta-analysis. Neurosurgery 73 : 193-199; discussion 199-200, 2013

2. Augsburger L, Farhat M, Reymond P, Fonck E, Kulcsar Z, Stergiopulos N, et al. : Effect of flow diverter porosity on intraaneurysmal blood flow. Klin Neuroradiol 19 : 204-214, 2009

3. Baek JW, Huh CW, Heo YJ, Yoo MW, Kwon SC, Kwon OK, et al. : Endovascular coiling of proximal middle cerebral artery aneurysms: is it safe and durable? Acta Neurochir (Wien) 160 : 2411-2418, 2018

4. Baráth K, Cassot F, Fasel JH, Ohta M, Rufenacht DA : Influence of stent properties on the alteration of cerebral intra-aneurysmal haemodynamics: flow quantification in elastic sidewall aneurysm models. Neurol Res 27 Suppl 1 : S120-S128, 2005

5. Bouzeghrane F, Naggara O, Kallmes DF, Berenstein A, Raymond J; International Consortium of Neuroendovascular Centres : In vivo experimental intracranial aneurysm models: a systematic review. AJNR Am J Neuroradiol 31 : 418-423, 2010

6. Briganti F, Napoli M, Tortora F, Solari D, Bergui M, Boccardi E, et al. : Italian multicenter experience with flow-diverter devices for intracranial unruptured aneurysm treatment with periprocedural complications--a retrospective data analysis. Neuroradiology 54 : 1145-1152, 2012

7. Brinjikji W, Murad MH, Lanzino G, Cloft HJ, Kallmes DF : Endovascular treatment of intracranial aneurysms with flow diverters: a meta-analysis. Stroke 44 : 442-447, 2013

8. Cebral JR, Mut F, Raschi M, Hodis S, Ding YH, Erickson BJ, et al. : Analysis of hemodynamics and aneurysm occlusion after flow-diverting treatment in rabbit models. AJNR Am J Neuroradiol 35 : 1567-1573, 2014

9. Chan TT, Chan KY, Pang PK, Kwok JC : Pipeline embolisation device for wide-necked internal carotid artery aneurysms in a hospital in Hong Kong: preliminary experience. Hong Kong Med J 17 : 398-404, 2011

10. Cirillo L, Dall'olio M, Princiotta C, Simonetti L, Stafa A, Toni F, et al. : The use of flow-diverting stents in the treatment of giant cerebral aneurysms: preliminary results. Neuroradiol J 23 : 220-224, 2010

11. Fernandez Zubillaga A, Guglielmi G, Viñuela F, Duckwiler GR : Endovascular occlusion of intracranial aneurysms with electrically detachable coils: correlation of aneurysm neck size and treatment results. AJNR Am J Neuroradiol 15 : 815-820, 1994

12. Ionita CN, Natarajan SK, Wang W, Hopkins LN, Levy El, Siddiqui AH, et al. : Evaluation of a second-generation self-expanding variable-porosity flow diverter in a rabbit elastase aneurysm model. AJNR Am J Neuroradiol 32 : 1399-1407, 2011

13. Kadirvel R, Ding YH, Dai D, Rezek I, Lewis DA, Kallmes DF : Cellular mechanisms of aneurysm occlusion after treatment with a flow diverter. Radiology 270 : 394-399, 2014

14. Kallmes DF, Altes TA, Vincent DA, Cloft HJ, Do HM, Jensen ME : Experimental side-wall aneurysms: a natural history study. Neuroradiology 41 : 338-341, 1999

15. Kallmes DF, Ding YH, Dai D, Kadirvel R, Lewis DA, Cloft HJ : A secondgeneration, endoluminal, flow-disrupting device for treatment of saccular aneurysms. AJNR Am J Neuroradiol 30 : 1153-1158, 2009

16. Kallmes DF, Hanel R, Lopes D, Boccardi E, Bonafé A, Cekirge $S$, et al. : International retrospective study of the pipeline embolization device: a multicenter aneurysm treatment study. AJNR Am J Neuroradiol 36 : 108-115, 2015

17. Kamran M, Yarnold J, Grunwald IQ, Byrne JV : Assessment of angiographic outcomes after flow diversion treatment of intracranial aneurysms: a new grading schema. Neuroradiology 53 : 501-508, 2011

18. Lee D, Yuki I, Murayama Y, Chiang A, Nishimura I, Vinters HV, et al. : Thrombus organization and healing in the swine experimental aneurysm model. Part I. A histological and molecular analysis. J Neurosurg 107 : 94-108, 2007

19. Lee R, Adlam D, Clelland CA, Channon KM : Lines of Zahn in coronary artery thrombus. Eur Heart J 33 : 1039, 2012

20. Li Z, Zhao R, Fang $X$, Zhou J, Jiang G, Huang Q, et al. : AMD3100 accelerates reendothelialization of neointima in rabbit saccular aneurysm after flow diverter treatment. World Neurosurg 107 : 416-423, 2017

21. Li ZF, Fang XG, Yang PF, Huang QH, Zhao WY, Liang C, et al. : Endothelial progenitor cells contribute to neointima formation in rabbit elastaseinduced aneurysm after flow diverter treatment. CNS Neurosci Ther $19: 352-357,2013$ 
22. Massoud TF, Guglielmi G, Ji C, Viñuela F, Duckwiler GR : Experimental saccular aneurysms. I. Review of surgically-constructed models and their laboratory applications. Neuroradiology 36 : 537-546, 1994

23. Meng H, Wang Z, Kim M, Ecker RD, Hopkins LN : Saccular aneurysms on straight and curved vessels are subject to different hemodynamics: implications of intravascular stenting. AJNR Am J Neuroradiol 27 : 1861-1865, 2006

24. Oh SY, Kim MJ, Kim BS, Shin YS : Treatment for giant fusiform aneurysm located in the cavernous segment of the internal carotid artery using the pipeline embolization device. J Korean Neurosurg Soc 55 : 32-35, 2014

25. Sadasivan C, Cesar L, Seong J, Rakian A, Hao Q, Tio FO, et al. : An original flow diversion device for the treatment of intracranial aneurysms: evaluation in the rabbit elastase-induced model. Stroke 40 : 952-958,
2009

26. Schafer Al : Genetic and acquired determinants of individual variability of response to antiplatelet drugs. Circulation 108 : 910-911, 2003

27. Szikora I, Berentei Z, Kulcsar Z, Marosfoi M, Vajda Z, Lee W, et al. : Treatment of intracranial aneurysms by functional reconstruction of the parent artery: the Budapest experience with the Pipeline embolization device. AJNR Am J Neuroradiol 31 : 1139-1147, 2010

28. Turk AS, Aagaard-Kienitz B, Niemann D, Consigny D, Rappe A, Grinde J, et al. : Natural history of the canine vein pouch aneurysm model. AJNR Am J Neuroradiol 28 : 531-532, 2007

29. Yu SC, Kwok CK, Cheng PW, Chan KY, Lau SS, Lui WM, et al. : Intracranial aneurysms: midterm outcome of pipeline embolization device--a prospective study in 143 patients with 178 aneurysms. Radiology 265 : 893-901, 2012 